\title{
CORRELATION BETWEEN ANISOTROPY DIRECTION AND PULSE SHAPE FOR METAL EVAPORATED TAPE
}

G. Krijnen 1, S. B. Luitjens 2, R.W. de Bie ${ }^{2}$, J.C. Lodder 1

1 University of Twente. P.O. Box 217, 7500 AE Enschede, The Netherlands.

2 Philips Research Laboratories. P.O. Box 80.000 . 5600 JA Eindhoven. The Netherlands.

\begin{abstract}
Measured replay pulses, recorded on Metal Evaporated tape, show a strong asymmetry and dependence on the direction of tape movement. It is found that pulse height and pulse width are unequal for both directions of tape movement. This is attributed to the non longitudinal easy-axis direction which causes differences in headtape geometry for both directions resulting in a different transition width. A simulation model is proposed. The differences in pulse asymmetry and pulse width between both directions of tape movement are very well reproduced by this model. From $X$-ray diffraction measurements and the results of the simulations it is concluded that not the columns but the crystallites act as magnetic entities for this kind of tape.
\end{abstract}

\section{INTRODUCTION}

Metal Evaporated (ME) tape is being investigated as a new medium for high density magnetic tape recording. Production of this tape according to the Contiruously Varying Incidence (CVI) method. yields a magnetic layer with an easy axis parallel to the incidence plane and tilted about 15-30 degrees out of the plane of the film. It is known that the recording properties of ME tape depend on the direction of tape movement relative to the recording head 1,2.3. Figure 1 shows the geometry of tape and recording head and the definition of $\mathrm{A}$ and $\mathrm{B}$ direction of tape movement.

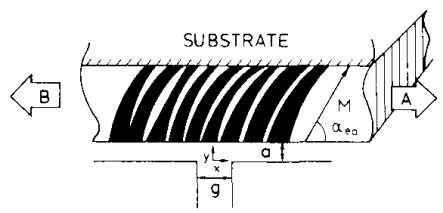

Figure 1: Schematic head geometry and definition of $\mathbf{A}$ and $\mathbf{B}$ direction of tape movement. $x$ is the angle between easy axis direction and film plane.

Several authors investigated the frequency response of $\mathrm{A}$ and $\mathrm{B}$ direction and found that the A-direction yields a higher output at short wavelength. $\mathrm{N}$. Ouchi et al. ${ }^{3}$ were able to clarify this phe nomenon by investigation of the component of the headfield parallel to the easy axis direction. It was shown that this component is varying during a write cycle in a different way for $\mathbf{A}$ and $\mathbf{B}$ direction resulting in a different magnetization. In this paper we report the investigations of readback-pulses for which both shape and amplitude depend on the direction of tape movement. Also a simulation model, which reproduces the asymmetric pulses and the differences for $\mathrm{A}$ and $\mathrm{B}$ direction very well, is presented.

\section{EXPERIMENTAL}

Structural properties. TEM micrographs of a cross section of the tape parallel to the coating direction, show a columnar structure with an average angle of 37 degrees relative to the plane of the film. The thickness of the magnetic film $(d)$ is $140 \mathrm{~mm}$. Auger Electron Spectroscopy (AES) revealed an average composition of $\mathrm{Co}_{77} \mathrm{Ni}_{10} \mathrm{O}_{13}$ at.\%. X-ray powder diffraction of the dissolved magnetic layer showed the presence of $\mathrm{Coh} / \mathrm{p}$ and NiOfic crystallites. The average grain size of the crystallites, as calculated from the halfwidth of the diffraction peaks, is about $30-50 \AA$ which implics that the columns are not single crystals but consist of many cristallites. Other authors reported same results 4 .

Magnetic properties. By means of a Vibrating Sample Magnetometer (VSM) hysteresis loops were measured for several angles between applied field and the filmplane by rotating the sample relative to the measuring direction. Figure 2 shows remanence and coercivity ver- sus angle. The easy axis direction $\left(x_{00}\right)$, estimated from the curve of the remanence, is 17 degrees relative to the filmplane. When compared to the average direction of the columns it is concluded that shape anisotropy of the columns is not the only source of the induced anisotropy in the film ${ }^{4}$. The saturation magnetization and maximum remanence were $5.3 \times 10^{2}$ and $4.3 \times 10^{2} \mathrm{kA} / \mathrm{m}$ respectively. The coercivity versus angle secms to follow a $1 / \cos$ dependence in the vicinity of the easy axis. As reported in 2.7 this behaviour is associated with domain nucleation and domain wall displacement. $H$ is $74 \mathrm{kA} / \mathrm{m}$ for $\alpha=\alpha_{e a}$ which is comparable to $H_{c}$. values reported by other authors 2.4 .

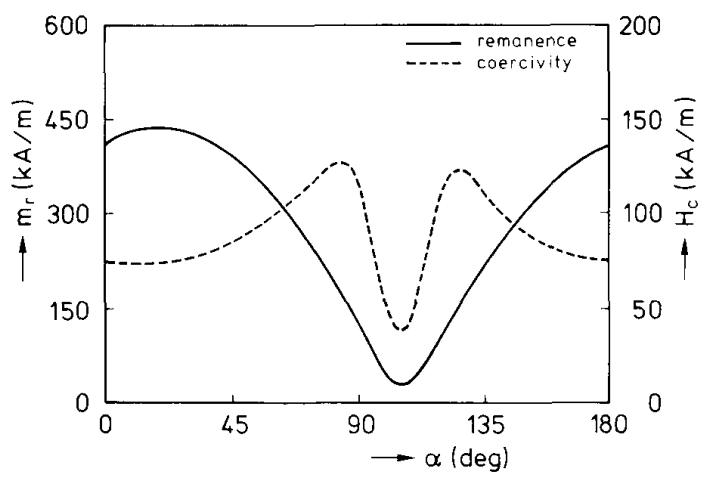

Figure 2: Remanence and coercivity versus the angle between applied field and filmplane.

Recording measurements. For recording measurements a drumhead configuration was used. 5 . Both reading and writing were performed with a ferrite head number of turns $N=16$, gaplength $g=0.26 \mu \mathrm{m}$. trackwidth $w=100 \mu \mathrm{m})$. The relative tape to head velocity (v) was $0.84 \mathrm{~m} / \mathrm{s}$. Medium and electronics noise were decreased by averaging 32 pulses for each direction. From experiments with reversed write and replay directions it was confirmed that the head behaved symmetrically. Measurement of the phase characteristic of the read amplifier showed some phase distortion at low frequencies for which the pulses were corrected. Figure 3 shows the measured pulses for $\mathrm{A}$ and $\mathrm{B}$ direction. Both directions show asymmetric pulses with a steep trailing edge for the A direction and a steep leading edge for the B direction. The pulse height $\left(V_{\text {pp }}\right)$ is 74 and 58 $m V$ (per turn per $m$ of trackwidth per $m / s$ of velocity) for $A$ and $B$ direction respectively.

\section{ANALYSIS}

Fourier transformation in the $k(=2 \pi / 2)$ domain provides a helpful tool for analyzing the measured pulses. Figure 4 shows the amplitude spectrum of a measured and a calculated pulse after Fourie transformation. From the slope of the amplitude spectrum the pulse width at half pulse height after phase equalization $\left(P W_{50}\right)$ can be calculated. $P W_{50}$ calculated according to this method contains the losses due to head to tape distance $(a)$. tape thickness $(d)$, gap loss and transition width $(c)$

The intercept of the extrapolated spectrum with the y-axis is pro portional to remanence. writedepth $(\delta)$ and read efficiency of the head $(\eta)$. The product of remanence and writedepth $\left(\delta, M_{r}\right)$ is cal culated taking $\eta=0.8$. The results of the analysis are summarized in table 1.

From table 1 it can be seen that $V_{a p}$ is smaller for the B direction. o. $M_{r}$ is nearly equal for both directions (especially when the accu- 
1818

\begin{tabular}{|c|c|c|c|}
\hline Direction & $V_{o p}$ & $P W_{50}$ & $\delta \cdot M_{r}$ \\
\hline A & $74 m V$ & $0.46 \mu m$ & $27 m A$ \\
\hline B & $58 m V$ & $0.53 \mu m$ & $24 m A$ \\
\hline
\end{tabular}

Table 1: Data of the measured pulses.

racy of the method is taken into account). This view is confirmed by other measurements. This implies that the write depth is equal for both directions. Therefore it is concluded that the higher $V_{o p}$ for the A direction is caused by a smaller transition width which is also reflected by the values of $P W_{50}$.
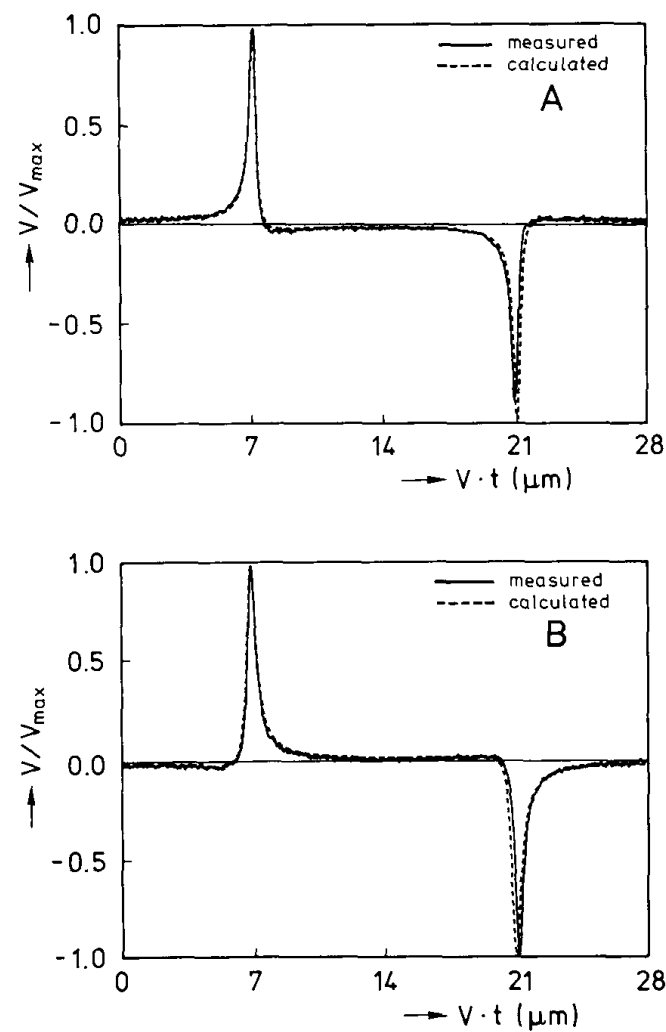

Figure 3: Measured and calculated pulses for tape movement in the A (upper figure) and B (lower figure) direction.

\section{SIMULATIONS}

Model. The following assumptions have been made to simulate readback pulses:

1. The magnetization is directed parallel to the easy axis $\left(\alpha_{e a}\right)$ over the entire trackwidth and thickness of the tape.

2. The magnetization has no z-component.

3. The shape and position of a transition are independent of $z$ over the trackwidth.

4. A transition has an arctangent shape. The position in $\mathrm{x}$ direction and the width of the transition are a function of the depth in the layer:

$$
\vec{M}(x, y)=\frac{2 M_{r}}{\pi} \arctan \left(\frac{x-\Delta x(y)}{c(y)}\right) \cdot \vec{e}_{e a}
$$

in which $c(y)$ is the transition width, $\Delta x(y)$ is the position of the

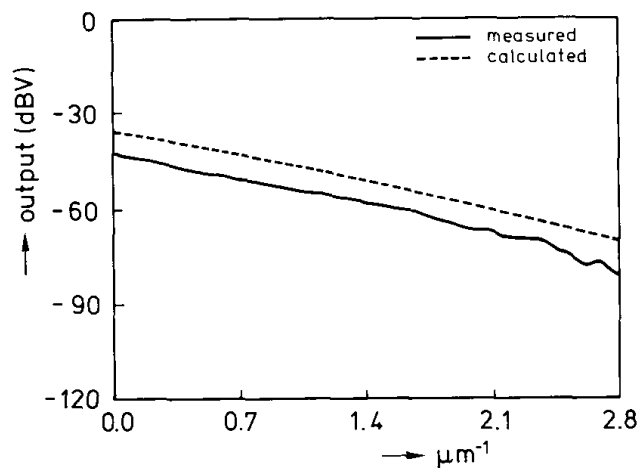

Figure 4: Amplitude spectrum of a measured pulse (solid line) and a simulated pulse (dashed lined) after Fourier transformation (A direction).

transition and $\vec{e}_{e a}$ is a unity vector in the direction of the easy axis.

5. $\Delta x(y)$ is calculated for a given $y$ by evaluating the projection of the headfield on the easy axis direction $\left(H_{e a}(x, y)\right)$ as a function of $x$ and by determining the position for which:

$$
\left|H_{e a}(x, y)\right|=\left|\vec{H}(x, y) \cdot \vec{e}_{e a}\right|=H_{c}
$$

$\vec{H}(x, y)$ in $(2)$ is the headfield for which the Karlquist expressions are used.

6. $c(y)$ is taken as the distance between the contours of $H_{e a}(x, y)=H_{c}$ and $H_{e a}(x, y)=2 H_{c}$ for given $y$ (see figure 5).

Note that assumptions 5 and 6 imply that a transition is not necessarily parallel to the columnar structure, i.e. the columns are not the magnetic entities in the film.

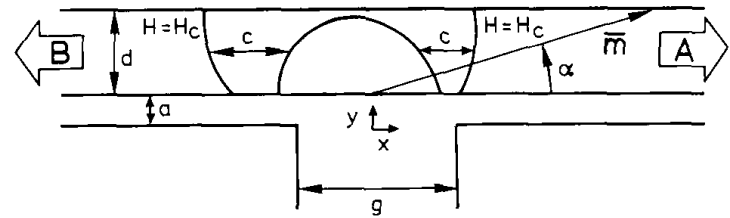

Figure 5: Calculated contours for $H_{e a}(x, y)=H_{c}$ and $H_{e a}(x, y)=2 H_{c}$. Note that a transition is written at the right hand side for tape movement in the A direction whereas it is written at the left hand side for the $B$ direction. The simulation was performed for a magneto motive force $(\eta I N)$ of $96 \mathrm{~mA}$ turns and a head to tape distance of $50 \mathrm{~nm}$.

Calculation of readback pulses is performed according to the following steps:

1. The magnetic film is divided in $m$ layers with a thickness $\Delta y=d / m$.

2. For each layer $\Delta x(y)$ and $c(y)$ are calculated.

3. The output of each layer is calculated in the frequency domain i.e. the coefficients of the Fourier series are determined.

4. After summation of the contributions of all layers the puise output is obtained by inverse Fourier transformation.

For steps 3 and 4 calculation is performed according to the following definitions:

$$
\begin{aligned}
& e(t)=\sum_{n=-\infty}^{n=+\infty} c_{n} e^{j n \omega_{0} t} \\
& c_{n}=\frac{1}{T_{0}} \int_{0}^{T_{0}} e(t) e^{-j n \omega_{0} t} d t .
\end{aligned}
$$

where $T_{0}$ is the period of the signal and $\omega_{0}=2 \pi / T_{n}$. Using the 
reciprocity theorem and the assumptions made before ( $3 \mathrm{~b}$ ) can be rewritten into:

$$
c_{n}=\mu_{0} w v \eta N \int_{a}^{a+d} \vec{H}\left(n k_{0}, v\right) \cdot j n k_{0} \dot{M}\left(-n k_{0}, v\right) d y
$$

in which $N$ is the number of turns, $w$ is the trackwidth, $v$ is the velocity and $k_{0}=2 \pi / v T_{0}$. Taking the Karlquist headtield equations and the Fourier series of (1) it follows for the $i^{\text {th }}$ layer that:

$$
\begin{aligned}
& c_{n i}=K_{n}\left(\cos x_{e a}-j \operatorname{sign}(n) \sin x_{e a}\right) e^{-\left|n k_{0}\right|\left(v_{i}+c\left(v_{i}\right) \mid\right.} e^{j n k_{0} \Delta x\left(y_{i}\right)} \\
& K_{n}=\mu_{0} w v \Delta y N \eta \frac{2 M_{r}}{\pi} k_{0} \frac{\sin \left(n k_{0} g / 2\right)}{n k_{0} g / 2} \frac{1-e^{-\left|n k_{0}\right| \Delta y}}{\left|n k_{0}\right| \Delta y} \quad n \neq 0
\end{aligned}
$$

where $y_{i}=a+i d / m(i=0,1,2 \ldots m-1)$ and $g$ is the gaplength. $c_{n}$ follows from the summation of the $c_{n i}$ of all $m$ layers. Substitution of $c_{n}$ into (3a) and rearranging $c_{n}$ for positive and negative $n$ yields:

$$
\begin{aligned}
& e(t)=\sum_{1}^{\infty} a_{n} \cos \left(n \omega_{0} t\right)+b_{n} \sin \left(n \omega_{0} t\right) \\
& a_{n}=2 K_{n} \sum_{i=0}^{m} \cos \left(\alpha_{e a}-n k_{0} \Delta x\left(y_{i}\right)\right) e^{\left.-n k_{0} \omega_{i}+c\left(y_{i}\right)\right)} \\
& b_{n}=2 K_{n} \sum_{i=0}^{m} \sin \left(\alpha_{e a}-n k_{0} \Delta x\left(y_{i}\right)\right) e^{-n k_{0}\left(b_{i}+c\left(v_{i}\right) t\right)}
\end{aligned}
$$

Results. Simulations have been performed using the values of $M_{r}, H_{c}, \alpha_{e a}, d$ as determined with VSM and TEM and $g, N, v$ and write current as in the recording experiments as described above. The head to tape distance, the number of layers $(m)$ and $\eta$ have been taken $50 \mathrm{~nm}, 50$ and 0.8 respectively. $k_{0}$ was aproximately equal as during the recording measurements. Figures 3,4 and 6 show the results of the simulation (dashed) compared to the measured pulses. From figures 3 and 4 it can be seen that the asymmetry and the $P W_{50}$ of the pulses are very well reproduced by the model. Nevertheless $V_{o p}$ and $\delta . M_{r}$ of the simulated pulses are larger which can be seen from figure 4 and table 2 .

\begin{tabular}{|c|c|c|c|}
\hline Direction & $V_{o p}$ & $P W_{50}$ & $\delta \cdot M_{r}$ \\
\hline A & $170 \mathrm{mV}$ & $0.42 \mu \mathrm{m}$ & $61 \mathrm{~mA}$ \\
\hline B & $144 \mathrm{mV}$ & $0.51 \mu \mathrm{m}$ & $61 \mathrm{~mA}$ \\
\hline
\end{tabular}

Table 2: Data of simulated pulses.

Figure 6 shows the calculated phase characteristic of both a measured and a simulated pulse (A direction) as obtained after Fourier transformation. The phase of the simulated pulse increases with decreasing wavelength like the phase of the measured pulse. This is caused by the write contours (as shown in figure 5) which can be seen from (6).

\section{CONCLUSIONS AND DISCUSSION}

Structural, magnetic and recording measurements on ME tape are reported. Small crystallites are observed by X-ray diffraction. The easy axis of the investigated tape was found 17 degrees tilted out of the filmplane. Measured readback pulses of ME tape show a dependence of shape and output on the direction of tape movement which are attributed to the non-longitudinal easy axis direction. It is found that the A direction yields higher maximum output and smaller $P W_{50}$, caused by a smaller transition width.

Simulations of readback pulses, including a write model, confirm this view. Figure 5 clearly illustrates that the gradient of $H_{e a}(x, y)$ is larger for the A direction resulting in sharper transitions. However, $V_{o p}$ and $\delta . M_{r}$ of the simulated pulses were found to be higher than for the measured pulses. $\dot{\partial} \cdot M_{r}$ of the measured pulses was lower than the value expected from magnetostatic measurements $(61 \mathrm{~mA})$.

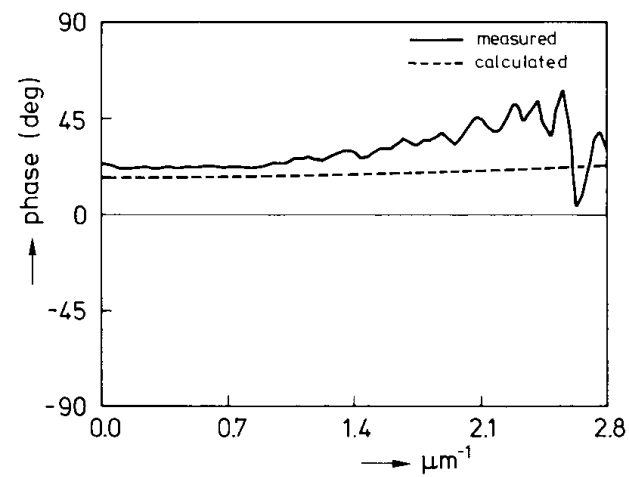

Figure 6: Phase characteristic of measured (solid line) and simulated (dashed line) pulses for the A direction of tape movement after Fourier transformation.

In order to clarify this discrepancy measurements with a metal in gap (MIG) type head ${ }^{8}$, with a high saturation magnetization, were carried out too. The results of these measurements were: $V_{o p}=119$ and $97 \mathrm{mV}$ for A and B direction respectively (nearly equivalent results were reported in 6 ). The corresponding values of $\delta . M$ were 47 and $46 m A$ for $\mathrm{A}$ and $\mathrm{B}$ direction respectively. This is in reasonable agreement with the $61 \mathrm{~mA}$ of the magnetostatic measurements if the uncertainty of $\eta$ is considered. From these experiments it is concluded that it is not possible to saturate the magnetic layer completely by means of a ferrite head due to the high coercivity of the medium. Use of a MIG head enables deeper writing. The discrepancy in $\delta \cdot M_{r}$ and $V_{o p}$ between the pulses as measured with a ferrite head and the simulated pulses is thought to arise from incomplete saturation of the magnetic layer which is not incorporated in the model.

In the calculation of the width and position of the transition no influence of demagnetizing fields were taken into account (except for the arctangent shape itself). Because the model predicts the transition width very well it is concluded that demagnetization does not play a significant role, i.e. the transition width is mainly determined by the coercivity of the film and the gradient of the headfield. Indeed, the transition width does not exceed the minimum transition width determined by the demagnetizing field, estimated from the measured $\delta . M_{r}$ and $H^{9}$

From the results of the simulation it is revealed that not the columns but the crystallites act as magnetic entities. Simulation of hysteresis loops, as reported in ? clectron diffraction studies as reported in 4 and the $x$-ray studies reported here seem to confirm this view.

Acknowledgement. We thank A. Rijckaert, E. de Niet, J. de Boer and B. Zwaans for the fruitful discussions and BASF who supplied the experimental samples for the investigations reported here.

Literature

' K. Ozawa et al.,

IEEF Trans. Magn. MAG-19, 1983, pp 1635-1637.

$2 \mathrm{~K}$. Shinohara et al.

IEEE Trans. Magn. MAG-20, 1984, pp 824-826.

${ }^{3}$ N. Ouchi et al., IEEE Trans. Magn. MAG-22, 1986, pp 385-387.

4 J.S. Gau et ai,, J. Appl. Phys. 61 (8), 1987, pp 3807-3809.

${ }^{5}$ S.B. Luitjens et al.

IEEE Trans. Magn. MAG-21, 1985, pp 1438-1440.

${ }^{6} \mathrm{~N}$. Watanabe et al.

IEEE Trans. Magn. MAG-21, 1985, pp 1368-1370.

${ }^{7}$ R.H. Victora, Phys. Rev. Let. 58 (17), 1987, pp 1788-1791.

8 J.J.M Ruigrok et al.,

IEEE Trans. Magn. MAG-20, 1984, pp 872-874

${ }^{9}$ C.D. Mee and E.D. Daniel (ed.).

Magnetic recording, Vol. 1, McGraw-Hill 1987, pp 63. 